\title{
11. RADIOLARIANS FROM THE EMPEROR SEAMOUNTS OF THE NORTHWEST PACIFIC: LEG 55 OF THE DEEP SEA DRILLING PROJECT
}

\author{
Hsin Yi Ling, Department of Geology, Northern Illinois University, DeKalb, Illinois 60115
}

\section{INTRODUCTION}

When the prospectus for Leg 55 was presented, it outlined two separate objectives: first, to drill into seamounts of the Emperor chain in the northwestern Pacific, and second, to recover sediments of the Mesozoic Pacific crust on the oceanic side of the Kurile Trench, east of Hokkaido, Japan. Accordingly, the purposes of my participation in the cruise were (1) to relate previous radiolarian zonation of high- and middle-latitude regions to that of the tropical Pacific; (2) to seek Mesozoic biostratigraphic succession in this far western corner of North Pacific; and (3) if possible, to relate this succession to that of land sections of Hokkaido. However, as the D/V Glomar Challenger finally sailed from Honolulu, Hawaii on 22 August 1977, after several postponements that delayed departure by more than one month, the shipboard scientists were belatedly informed that the drilling for Mesozoic crust was cancelled. Consequently, the scope of this report is necessarily more limited than had been anticipated.

Methods of laboratory treatment of the cored sediments, and of recording the illustrated specimens in the strewn slides with an application of England Finder, are essentially the same as those of the previous reports (Ling, 1973, 1975, 1979). Relative abundance (A, abundant; C, common; F, frequent, $\mathrm{R}$, rare) and state of preservation (G, good; $F$, fair; $P$, poor) are repeated according to the shipboard paleontological format.

All the slides examined during the study discussed herein will be deposited permanently in the micropaleontological collection, Department of Oceanography, University of Washington, Seattle, Washington.

\section{RADIOLARIAN OCCURRENCES AT EACH SITE}

Throughout the entire cruise, drillings were attempted on top of four seamounts (Figure 1, Table 1), and the recovery of soft sediments was disappointingly low. The percentage of recovery solely for soft sediments was merely 32.6 per cent after the recalculation, instead of 51.0 per cent for the total recovery (Jackson, Koizumi et al., 1978).

The biostratigraphic zonation proposed by Hays (1970) and Foreman (1975) was employed throughout the cruise and during the present investigation.

\section{Site $\mathbf{4 3 0}$}

Site 430 is on top of Ōjin Seamount, and three holes were drilled into what appeared to be a lagoonal sediment pond. Radiolarians are completely absent from the sediments of Hole 430; sediment consists of Recent to Eocene pebbly mudstone. Sediments from Hole 430A

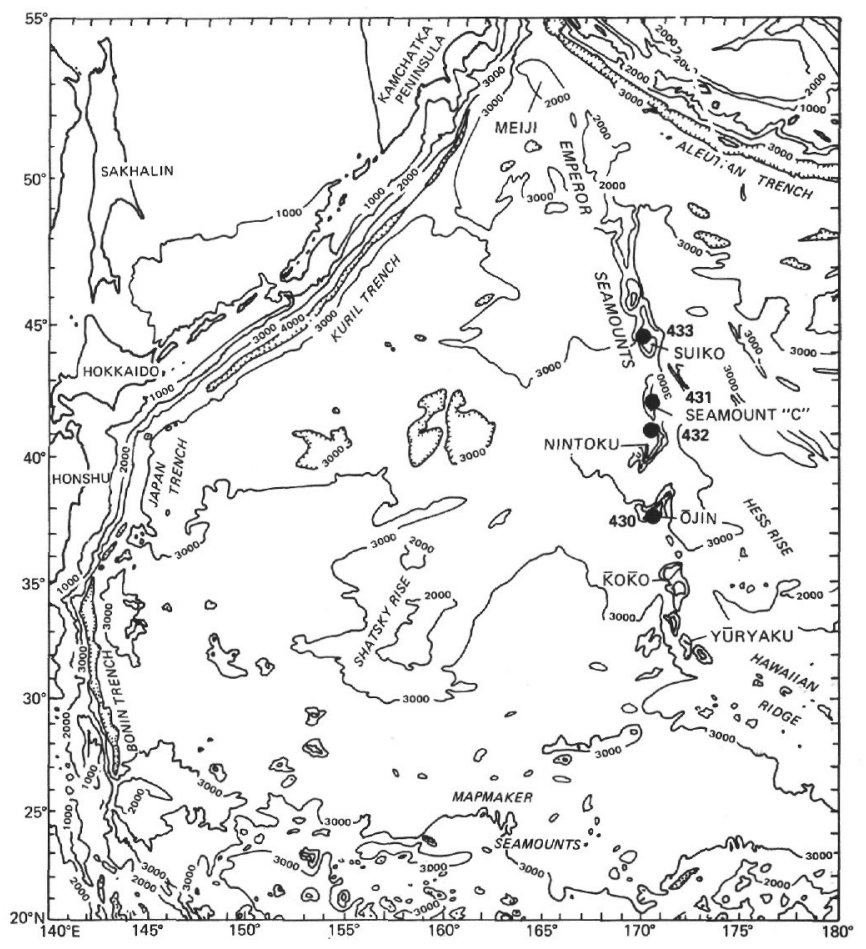

Figure 1. Map of the northwestern Pacific Ocean showing sites drilled during Leg 55 of Deep Sea Drilling Project.

are upper Paleocene through upper Eocene calcareous ooze and volcanic ash and sand. Only the soft surface sediments of Hole 430B yielded abundant and wellpreserved radiolarians of a transitional Quaternary assemblage.

\section{Site 431}

Site 431 is on a faulted terrace on the east side of the newly postulated Yōmei Seamount (Dalrymple, personal communication). Coring was attempted twice for the surface sediments at Hole 431, and only moderately well-preserved Recent radiolarians, few to rare, were recovered. Sediments from Core 1 ( 0 to $2 \mathrm{~m}$ ) and the uppermost part of Core $2(7.5 \mathrm{~m})$ of Hole $431 \mathrm{~A}$ contain rare, moderately well preserved middle Pleistocene radiolarians of the Stylatractus universus Zone. The calcareous sediments from Sample 2, CC are Eocene, however, and radiolarians are completely absent.

\section{Site $\mathbf{4 3 2}$}

Two holes were drilled on the presumed perched terrace deposits on the top of Nintoku Seamount, but only Hole 432A, (Core 1 [0 to $3 \mathrm{~m}$ ]) contained common to 
TABLE 1

Coordinates of Drilling Sites and Coring Summary of DSDP Leg 55

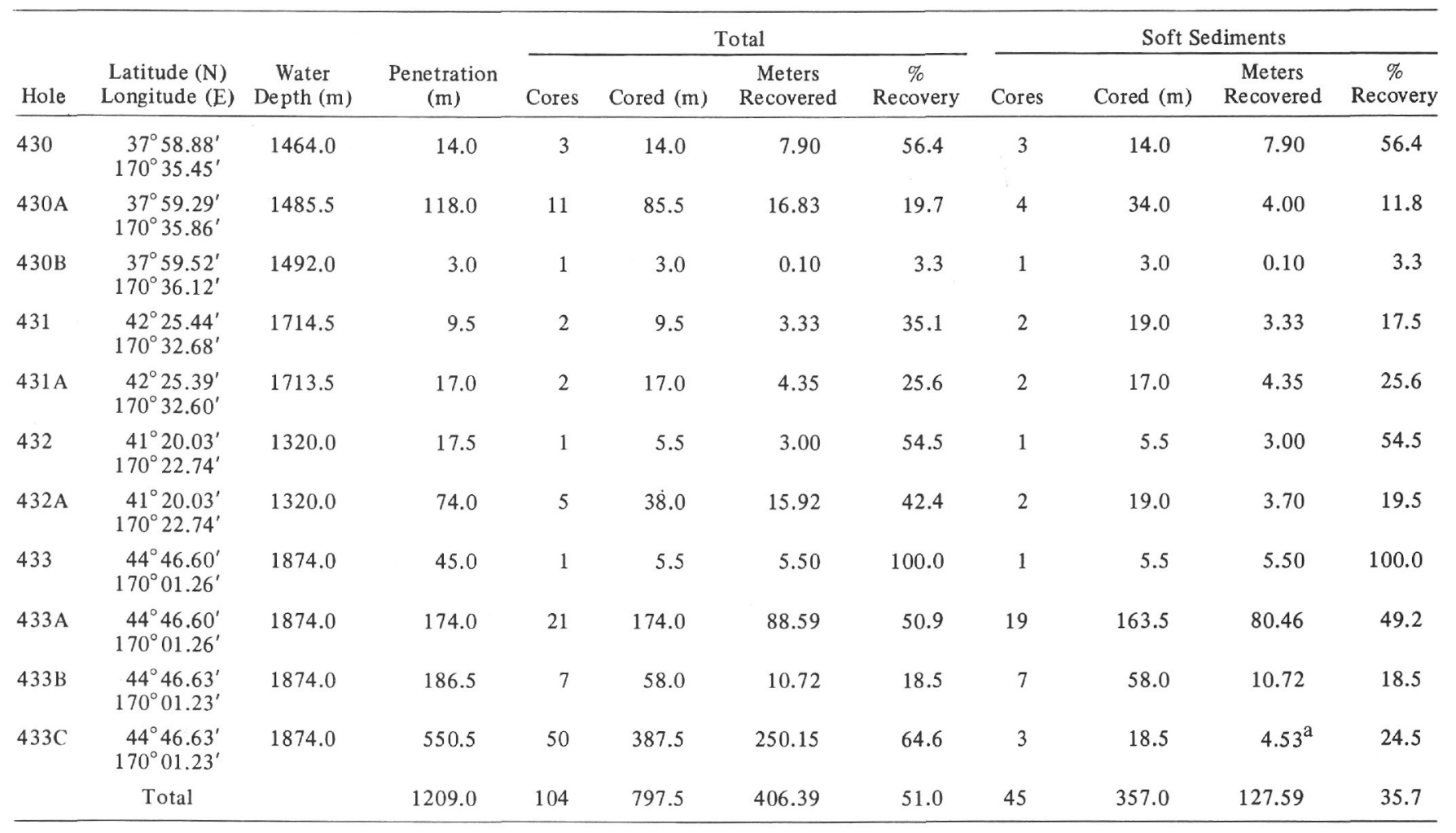

aReef carbonate sands sandwiched between the flows.

rare and well-preserved to moderately well-preserved radiolarians of a modern transitional assemblage.

\section{Site 433}

Throughout the entire cruise, abundant well-preserved radiolarians were recovered only from this reentry site, located in a complexly deformed marginal structural basin associated with a fairly extensive lagoonal complex on Suiko Seamount.

Within 5 meters of the drilled sediments of Hole 433, three radiolarian zones were recognized: Sample 1-1, $75-77 \mathrm{~cm}(0.7 \mathrm{~m})$, Botryostrobus auritusaustralis Zone; Sample 1-2, 10-13 cm (1.6 m), Stylatructus universus Zone; and 1-2, 113-115 cm (2.6 m), Lamprocyrtis heteroporos Zone (Table 2). Thus, at least the entire lower Pleistocene Eucyrtidium matuyamai Zone, and also probably the upper part of the upper Pliocene Lamprocyrtis heteroporos Zone were missing. This seems to correspond to a lithologic change from the sandy diatom-nannofossil ooze to diatom-nannofossil ooze below.

Hole 433A was the most productive for radiolarians (Table 3). Continuous coring recovered sediments containing the following zones before this group of siliceous microfossils vanished starting with Core 8,62 meters from the sea floor: Sample 1-1, 4-13 cm (about 0.1 $\mathrm{m})$, Stylatractus universus Zone; Sample 1-1, 23-25 cm $(0.25 \mathrm{~m})$ to Sample 3, CC (24 m), probably Sphaeropyle langii Zone; Sample 4-1, 13-15 cm (about $24.1 \mathrm{~m}$ ) to Sample 6-6, 10-12 cm (50.6 m), Stichocorys peregrina Zone; Sample 6-6, 140-142 cm (51.9 m) to Sample 7,
CC (62 m), unzoned middle Miocene section characterized by the occurrence of Cyrtocapsella tetrapera, Lychnocanomma elongata, and Eucyrtidium sp.

Soft sediments and reef carbonate sands from Hole 433B, Cores 1 through 4, and Hole 433C, Core 3 within basaltic flows, failed to yield radiolarians.

\section{DISCUSSION}

Although Leg 55 recovered only a small amount of radiolarian-bearing sediments, geological occurrence of the following radiolarians bears potential significance for future consideration of regional biostratigraphy in this part of the North Pacific Ocean:

1. There was a slight overlap after the initial appearance of Lamprocyrtis heteroporos and before the last appearance of Stichocorys peregrina. In Hole 433A, this is represented in Samples 4-1, 13-15 cm and 4-2, 44-46 $\mathrm{cm}$.

2. The uppermost occurrence of Cyrtocapsella tetrapera is in Sample 6-6, 140-142 cm, in the middle Miocene unzoned section which is Denticula lauta (diatom) Zone (Koizumi, personal communication). Apparently, such occurrence has been widely recognized in the North Pacific (Ling, 1973), North Atlantic (Ling, 1979), and Antarctic (Bandy et al., 1971).

3. The occurrence of Lychnocanomma elongata, although only a few specimens were found (from Hole 433A), marks the northernmost occurrence for the species in the North Pacific. 
TABLE 2

Radiolarian Species Distribution, Abundance, and Preservation, Site 433

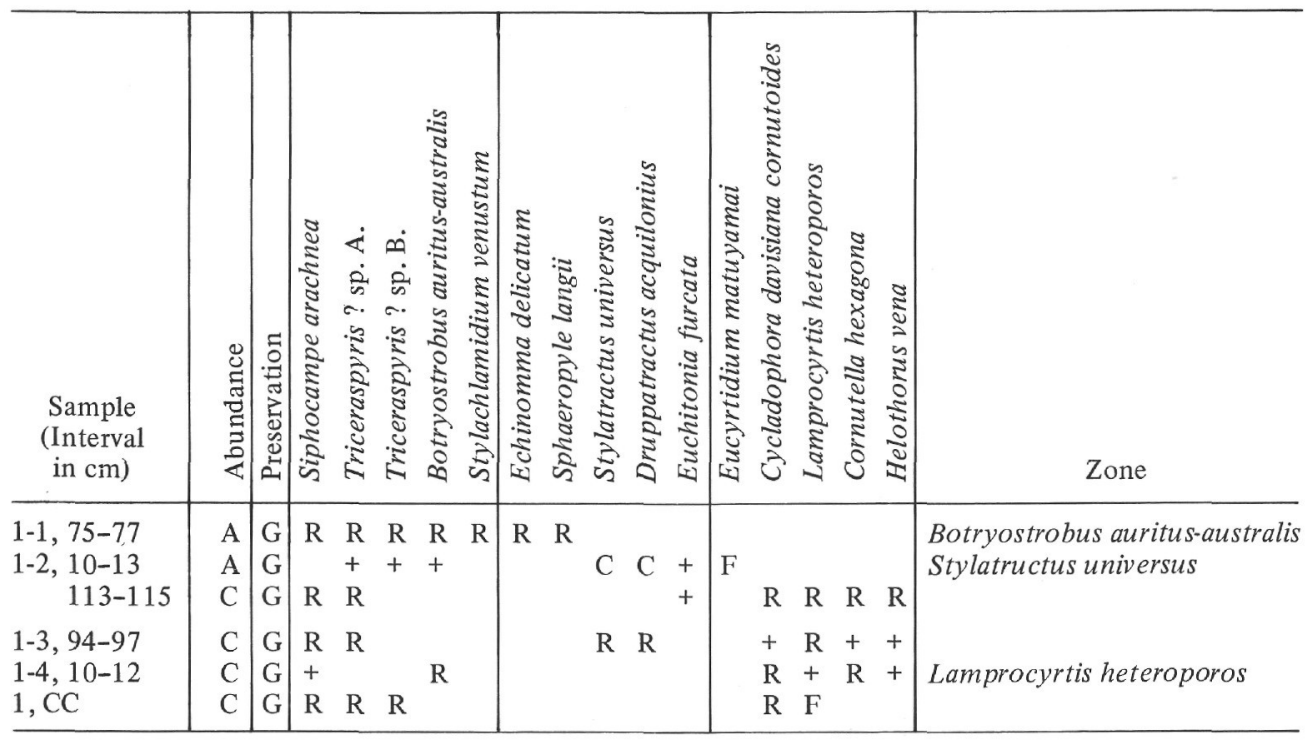

Note: $\mathrm{A}=$ abundant $; \mathrm{C}=\operatorname{common} ; \mathrm{F}=$ frequent $\mathrm{R}=\operatorname{rare} ;+=\operatorname{single}$ specimen $; \mathrm{G}=\operatorname{good}$.

4. The occurrence of Cannartus violina and C. prismaticus in the lower part of Core 6 marks their northernmost occurrence. Although absence of many co-occurring warm-water index taxa make the precise age and/or zonal assignments for the sediments impossible at this time, a middle Miocene assignment seems plausible.

\section{RADIOLARIAN FAUNAL LIST}

Following is an alphabetical listing of radiolarian species recognized in the examined sediments. Most of the taxa have been described or discussed adequately during the past several years, and references are readily available. Readers are therefore directed to consult such publications.

Anthocyrtidium ophirense (Ehrenberg), Nigrini, 1967, pp. 56-58, pl. 6, figure 3 = Anthocyrtis ophirensis Ehrenberg, 1872, p. 301 . (Plate 2, figure 14).

Artostrobus annulatus (Bailey), Haeckel, 1887, p. 1481 = Cornutella? annulata Bailey, 1856, p. 3, pl. 1, figure 5a, b.

Botryostrobus aquilonaris (Bailey), Nigrini, 1977, p. 246, pl. 1, figure $1=$ Eucyrtidium aquilonaris Bailey, 1856, p. 4, pl. 1, figure 9. (Plate 2, figure 20).

Botryostrobus auritus-australis (Ehrenberg) group, Nigrini, 1977, pp. 246, 248, pl. 1, figures $2-5=$ Lithocampe lineata Ehrenberg, 1838 , p. 130 (partim).

Cannartus prismaticus (Haeckel), Riedel and Sanfilippo, 1970, p. 520, pl. 15, figure $1 .=$ Pipettella prismatica Haeckel, 1887, p. 305. (Plate 1, figure 3).

Cannartus violina Haeckel, 1887, p. 358, pl. 39, fig. 10. (Plate 1, figure 4).

Carpocanistrum sp. A., Ling, 1973, p. 730, pl. 12, figure 3 (Plate 2, figure 18).

Carpocanistrum sp. C., Ling, 1973, p. 730, pl. 12, figure 5 (Plate 2, figure 19).

Clathrocyclas craspedota (Jorgensen), Jorgensen, 1905, p. 139, pl. 17, figures 98-100. = Theocalyptra craspedota Jorgensen, 1900, p. 85. (Plate 2, figure 2).

Cornutella hexagona Haeckel, 1887, p. 1180, pl. 54, figure 9.

Cornutella profunda Ehrenberg, 1859, p. $31=$ C. clathrata? profunda Ehrenberg, 1856, pl. 35B, figure 21.
Cycladophora davisiana davisiana Ehrenberg, Petrushevskaya, 1967, pp. 122-124, figure $69,1-7=$ Cycladophora? davisiana Ehrenberg, 1862, p. 297.

Cycladophora davisiana cornutoides (Petrushevskaya), Kling, 1977, p. 217, pl. 1, figure 20. (Plate 2, figure 1).

Cyrtocapsella cornuta Haeckel, 1887, p. 1513, pl. 78, figure 9.

Cyrtocapsella japonica (Nakaseko), Sanfilippo and Riedel, 1970, p. 452, pl. 1, figures 13-15 = Eusyringium japonicum Nakaseko, 1963, p. 193, pl. 4, figures 1-3. (Plate 2, figure 4).

Cyrtocapsella tetrapera Haeckel, Sanfilippo and Riedel, 1970, p. 453, pl. 1, figures 16-18 = Cyrtocapsa tetrapera Haeckel, 1887, p. 1512, (Plate 2, figure 5).

Cyrtocapsella sp. Remarks: Three-segmented, triangular form, with the maximum width near the middle of abdominal segment. Length of thorax is approximately equal to that of abdomen. The abundant but limited occurrence, only in the lowest part of Core 6 from Hole 433A just below the extinction level of Cyrtocapsella tetrapera, may suggest the possible biostratigraphic significance for the species in the western North Pacific. (Plate 2, figure 6).

Dictyocryphallus papillosus (Ehrenberg), Nigrini, 1967, pp. 63, 64, p. 6, figure $6=$ Eucyrtidium papillosum Ehrenberg, 1872, p. 310 .

Druppatractus acquilonius Hays, 1970, p. 214, pl. 1, figures 4-5. (Plate 1, figure 1).

Echinomma delicatum (Dogiel), Petrushevskaya, 1967, pp. 18-20, figure 11 = Heliosoma delicatum Dogiel and Reshetnyak, 1952, pp. 7, 8. Fig. 2.

Euchitonia furcata Ehrenberg, 1861, p. 767. (Plate 1, figure 6).

Eucyrtidium calvertense Martin, 1904, p. 450, pl. 130, figure 5. (Plate 2, figure 7).

Eucyrtidium matuyamai Hays, 1970, p. 213, pl. 1, figures 7-9. (Plate 2, figure 8).

Eucyrtidium sp., Ling, 1973, p. 778, pl. 1, figures 9-10. Remarks: The specimens observed here belong to the same taxon recognized from Site 183 of eastern North Pacific, indicating the wide geographic occurrence of this taxon. (Plate 2, figure 9).

Heliodiscus asteriscus Haeckel, 1887, p. 445, pl. 33, figure 8. (Plate 1, figure 5).

Helotholus vema Hays, 1965, p. 176, pl. II, figure 3. (Plate 1, figure 11).

Lamprocyrtis heteroporos (Hays), Kling, 1973, p. 639, pl. 5, figures 19, 20 = Lamprocyclas heteroporos Hays, 1965, pp. 179, 180, pl. III, figure 1 (Plate 2, figure 17).

Lamprocyclas maritalis maritalis Haeckel, 1887, p. 1390, pl. 74, figures 3, 14. (Plate 2, figure 15).

Lamprocyclas sp. (Plate 2, figure 16). 
TABLE 3

Radiolarian Species Distribution, Abundance, and Preservation, Hole 433A

\begin{tabular}{|c|c|c|c|c|c|c|c|c|c|c|c|c|}
\hline $\begin{array}{c}\text { Sample } \\
\text { (Interval } \\
\text { in } \mathrm{cm} \text { ) }\end{array}$ & & & 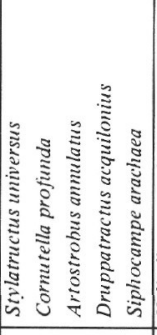 & 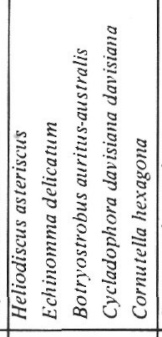 & 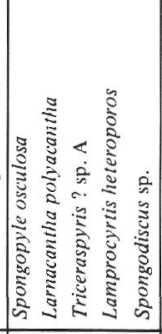 & 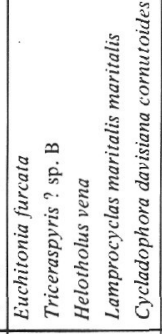 & 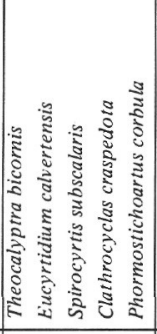 & 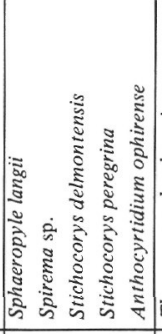 & 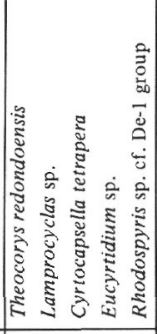 & 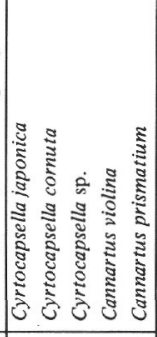 & 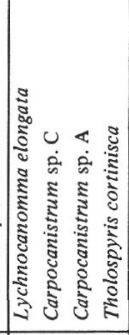 & Zone \\
\hline $\begin{array}{c}1-1,4-13 \\
23-25 \\
70-73 \\
133-135 \\
1, \mathrm{CC} \\
\end{array}$ & $\begin{array}{l}\mathrm{F} \\
\mathrm{A} \\
\mathrm{A} \\
\mathrm{A} \\
\mathrm{A}\end{array}$ & \begin{tabular}{|l|}
$\mathrm{M}$ \\
$\mathrm{G}$ \\
$\mathrm{G}$ \\
$\mathrm{G}$ \\
$\mathrm{G}$ \\
\end{tabular} & $\begin{array}{|ccccc|}+ & \mathrm{R} & \mathrm{R} & \mathrm{F} & \mathrm{C} \\
\mathrm{F} & & & \mathrm{A} & \mathrm{R} \\
\mathrm{R} & \mathrm{R} & + & \mathrm{R} & \mathrm{R} \\
\mathrm{R} & \mathrm{R} & \mathrm{R} & \mathrm{R} & \mathrm{C} \\
\mathrm{F} & & \mathrm{F} & & \mathrm{F} \\
\end{array}$ & $\begin{array}{|ccccc|}+ & \mathrm{F} & \mathrm{F} & \mathrm{R} & \mathrm{R} \\
\mathrm{R} & \mathrm{C} & \mathrm{F} & + \\
& \mathrm{F} & \mathrm{R} & + \\
& \mathrm{R} & \mathrm{R} & \mathrm{R} \\
& & & \mathrm{R} \\
\end{array}$ & $\begin{array}{lllll}\mathrm{R} & \mathrm{F} & \mathrm{R} & \mathrm{R} & \mathrm{R} \\
& \mathrm{R} & \mathrm{F} & \mathrm{R} \\
+ & + & \mathrm{F} & \mathrm{F} \\
\mathrm{F} & \mathrm{R} & \mathrm{C} & \mathrm{C} \\
\end{array}$ & $\begin{array}{|lllll|}R & R & F & F & \\
& C & F & & R \\
& & & & F \\
\end{array}$ & & & & & & \multirow{3}{*}{ Sphaeropyle langii } \\
\hline $\begin{array}{l}2-1,32-34 \\
125-127 \\
2-2,32-34 \\
2, \mathrm{CC} \\
3-1,13-15 \\
\end{array}$ & $\begin{array}{l}\mathrm{A} \\
\mathrm{A} \\
\mathrm{A} \\
\mathrm{C} \\
\mathrm{A}\end{array}$ & \begin{tabular}{|l|}
$G$ \\
$G$ \\
$G$ \\
$G$ \\
$G$ \\
\end{tabular} & $\begin{array}{|llll|}\mathrm{F} & & \mathrm{A} & \mathrm{R} \\
\mathrm{F} & + & + & \mathrm{F} \\
\mathrm{R} & & \mathrm{F} \\
\mathrm{F} & \mathrm{R} & \mathrm{C} \\
\mathrm{R} & \mathrm{F} & \mathrm{F} \\
\mathrm{R} & \mathrm{R} & \mathrm{F} \\
\end{array}$ & \begin{tabular}{|llll}
$\mathrm{R}$ & $\mathrm{R}$ & $\mathrm{F}$ & \\
+ & $\mathrm{F}$ & $\mathrm{R}$ & \\
+ & $\mathrm{F}$ & $\mathrm{F}$ & \\
& & $\mathrm{R}$ & $\mathrm{F}$ \\
& $\mathrm{F}$ & $\mathrm{F}$ & \\
\end{tabular} & $\begin{array}{|lllll|}\mathrm{R} & \mathrm{C} & \mathrm{R} & & \\
\mathrm{R} & \mathrm{R} & \mathrm{R} & \mathrm{F} & \mathrm{R} \\
\mathrm{R} & \mathrm{F} & \mathrm{R} & \mathrm{F} & \mathrm{F} \\
\mathrm{F} & & \mathrm{C} & \mathrm{C} & \\
+ & \mathrm{R} & \mathrm{R} & \mathrm{F} & \\
\end{array}$ & \begin{tabular}{|rrrr} 
& & & \\
+ & $R$ & $R$ & $R$ \\
$F$ & + & + \\
& & + & $R$ \\
& $R$ & & $R$ \\
\end{tabular} & 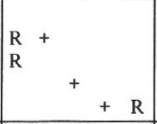 & & & & & \\
\hline $\begin{array}{l}3-2,13-15 \\
3-3,13-15 \\
3-4,23-25 \\
3-5,13-15 \\
3-6,13-15 \\
\end{array}$ & \begin{tabular}{l|}
$\mathrm{A}$ \\
$\mathrm{A}$ \\
$\mathrm{A}$ \\
$\mathrm{A}$ \\
$\mathrm{A}$
\end{tabular} & \begin{tabular}{|l|}
$\mathrm{G}$ \\
$\mathrm{G}$ \\
$\mathrm{G}$ \\
$\mathrm{G}$ \\
$\mathrm{G}$ \\
\end{tabular} & $\begin{array}{|lllll|}+ & \mathrm{R} & + & \mathrm{R} & \mathrm{F} \\
+ & & + & + & \mathrm{R} \\
\mathrm{R} & & & \mathrm{R} & \mathrm{F} \\
\mathrm{R} & \mathrm{R} & & + & \mathrm{R} \\
\mathrm{R} & \mathrm{R} & & \mathrm{R} & \mathrm{R} \\
\end{array}$ & $\begin{array}{|llll|}\mathrm{R} & \mathrm{R} & \mathrm{F} & \mathrm{R} \\
\mathrm{R} & \mathrm{R} & \mathrm{C} & \mathrm{R} \\
+ & \mathrm{R} & \mathrm{F} & \mathrm{R} \\
\mathrm{R} & \mathrm{R} & \mathrm{F} & \\
\mathrm{R} & \mathrm{R} & \mathrm{F} & \\
\end{array}$ & $\begin{array}{llll} & \mathrm{R} & \mathrm{F} & \mathrm{F} \\
+ & \mathrm{R} & \mathrm{R} & \mathrm{F} \\
\mathrm{R} & \mathrm{R} & \mathrm{F} & \mathrm{F} \\
& \mathrm{R} & \mathrm{R} & \mathrm{F} \\
\mathrm{R} & \mathrm{R} & \mathrm{F} & \mathrm{F}\end{array}$ & \begin{tabular}{|rrr}
$\mathrm{R}$ & $\mathrm{R}$ & $\mathrm{R}$ \\
+ & + & \\
$\mathrm{R}$ & \\
+ & $\mathrm{R}$ & \\
& $\mathrm{R}$ & $\mathrm{R}$ \\
\end{tabular} & 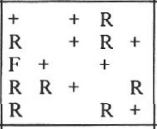 & + & & & & \\
\hline $\begin{array}{l}3, \mathrm{CC} \\
4-1,13-15 \\
4-2,5-7 \\
44-46 \\
4-3,10-12 \\
\end{array}$ & \begin{tabular}{l|}
$\mathrm{A}$ \\
$\mathrm{A}$ \\
$\mathrm{A}$ \\
$\mathrm{A}$ \\
$\mathrm{A}$
\end{tabular} & \begin{tabular}{|l|}
$\mathrm{G}$ \\
$\mathrm{G}$ \\
$\mathrm{G}$ \\
$\mathrm{G}$ \\
$\mathrm{G}$ \\
\end{tabular} & $\begin{array}{|lllll|} & \mathrm{F} & \mathrm{R} & & \mathrm{C} \\
\mathrm{F} & + & & \mathrm{R} & \\
\mathrm{R} & & & \mathrm{R} & \mathrm{R} \\
\mathrm{F} & \mathrm{R} & & \mathrm{F} & \mathrm{R} \\
& & & & \mathrm{R} \\
\end{array}$ & $\begin{array}{rrr} & & \\
& \mathrm{F} & \mathrm{R} \\
+ & \mathrm{R} & \\
\mathrm{R} & & \\
+ & & + \\
\end{array}$ & \begin{tabular}{|cccc} 
& $R$ & $F$ & $F$ \\
& $C$ & & $R$ \\
+ & $R$ & & $R$ \\
& $R$ & & $R$ \\
& $R$ & & \\
\end{tabular} & $\begin{array}{l}\mathrm{R}^{+} \\
+ \\
\mathrm{R}\end{array}$ & $\begin{array}{|lll|} & & \mathrm{R} \\
\mathrm{R} & \mathrm{R} & \mathrm{R} \\
+ & \mathrm{R} & \mathrm{R} \\
& + & \\
& + & \end{array}$ & 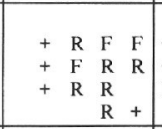 & $\begin{array}{l}++ \\
+ \\
F\end{array}$ & & & \multirow{5}{*}{ Stichocorys peregrina } \\
\hline $\begin{array}{l}4-4,10-12 \\
4-5,10-12 \\
4-6,10-12 \\
4, \mathrm{CC} \\
5-1,10-12\end{array}$ & \begin{tabular}{l|}
$\mathrm{A}$ \\
$\mathrm{A}$ \\
$\mathrm{A}$ \\
$\mathrm{A}$ \\
$\mathrm{A}$
\end{tabular} & \begin{tabular}{|l|}
$G$ \\
$G$ \\
$G$ \\
$G$ \\
$G$ \\
\end{tabular} & $\begin{array}{|llll|} & + & & \\
+ & & & \\
+ & + & & \\
F & \mathrm{~F} & \mathrm{C} & \\
+ & \mathrm{C} & & \\
\end{array}$ & $+R$ & $\begin{aligned} & R \\
& \mathrm{~F} \\
&+ \mathrm{F} \\
& \mathrm{F} \\
&\end{aligned}$ & $\begin{array}{ll}R & \\
R & \\
+ & F\end{array}$ & $\begin{array}{lll}\mathrm{R}+ & \\
+ & & \\
& + \\
& \mathrm{F}\end{array}$ & 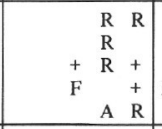 & F & & & \\
\hline $\begin{array}{r}5-2,10-12 \\
5-3,10-12 \\
5-4,10-12 \\
60-62 \\
5-5,10-12 \\
\end{array}$ & \begin{tabular}{l|}
$\mathrm{A}$ \\
$\mathrm{A}$ \\
$\mathrm{A}$ \\
$\mathrm{A}$ \\
$\mathrm{A}$
\end{tabular} & \begin{tabular}{|l|l}
$G$ \\
$G$ \\
$G$ \\
$G$ \\
$G$ \\
\end{tabular} & \begin{tabular}{|lll}
+ & $\mathrm{R}$ & $\mathrm{R}$ \\
+ & & \\
+ & $\mathrm{R}$ \\
+ & $\mathrm{R}+$ & \\
+ & + & \\
+ & + & $\mathrm{R}$ \\
\end{tabular} & + & $\begin{array}{l}\text { A } \\
\text { A } \\
\text { A }\end{array}$ & & $\begin{array}{l}+ \\
\mathrm{R}\end{array}$ & $\begin{array}{rr}+ & \mathrm{A} \\
+ & \mathrm{A} \\
& \mathrm{A} \\
& \mathrm{F} \\
+\quad \mathrm{F} & \mathrm{F} \\
\end{array}$ & & & & \\
\hline $\begin{array}{l}5-6,10-12 \\
5, \mathrm{CC} \\
6-1,10-12 \\
6-2,10-12 \\
6-3,10-12 \\
\end{array}$ & \begin{tabular}{l|}
$\mathrm{A}$ \\
$\mathrm{A}$ \\
$\mathrm{A}$ \\
$\mathrm{A}$ \\
$\mathrm{A}$
\end{tabular} & & \begin{tabular}{|lll}
$R$ & + \\
$F$ & $R$ & \\
$R$ & $R$ & + \\
$R$ & +
\end{tabular} & $\begin{array}{r}\mathrm{R}+ \\
+ \\
\mathrm{R} \\
+\quad \mathrm{R} \\
\end{array}$ & $\mathrm{R}$ & + & $\begin{array}{l}\mathrm{R} \\
\mathrm{R}\end{array}$ & $\begin{array}{r}+R \\
+\quad R \\
+R \\
\\
R\end{array}$ & & & & \\
\hline $\begin{array}{r}6-4,10-12 \\
80-82 \\
6-5,10-12 \\
90-92 \\
6-6,10-12 \\
\end{array}$ & \begin{tabular}{l|}
$\mathrm{C}$ \\
$\mathrm{A}$ \\
$\mathrm{F}$ \\
$\mathrm{C}$ \\
$\mathrm{C}$
\end{tabular} & \begin{tabular}{l|l}
$G$ \\
$G$ \\
$G$ \\
$G$ \\
$G$ \\
\end{tabular} & \begin{tabular}{|ll} 
& $\mathrm{R}$ \\
$\mathrm{R}+$ & + \\
$\mathrm{R}$ & \\
\end{tabular} & $\begin{array}{l}+ \\
+ \\
+ \\
+ \\
+ \\
+\end{array}$ & & + & $\mathrm{R}^{+}$ & $\begin{array}{l}\mathrm{R} \\
+ \\
\mathrm{R} \\
+ \\
\end{array}$ & & & & \\
\hline $\begin{array}{l}140-142 \\
6-7,47-49 \\
6, \mathrm{CC} \\
7-1,10-12 \\
80-82 \\
\end{array}$ & \begin{tabular}{l|}
$\mathrm{A}$ \\
$\mathrm{A}$ \\
$\mathrm{C}$ \\
$\mathrm{A}$ \\
$\mathrm{C}$
\end{tabular} & \begin{tabular}{l|l}
$G$ \\
$G$ \\
$M$ \\
$G$ \\
$M$
\end{tabular} & $\begin{array}{ll}\mathrm{R} & \mathrm{R} \\
& \\
+ & + \\
\mathrm{R} & \mathrm{R} \\
\end{array}$ & $\begin{array}{r}+ \\
+\quad \mathrm{R} \\
\end{array}$ & + & $\mathrm{R}$ & $\mathrm{R}$ & $\begin{array}{l}+ \\
\mathrm{R} \\
\end{array}$ & \begin{tabular}{lll|}
$\mathrm{F}$ & $\mathrm{R}$ & $\mathrm{R}$ \\
$\mathrm{C}$ & $\mathrm{R}$ & \\
$\mathrm{A}$ & $\mathrm{R}$ \\
$\mathrm{A}$ & + & \\
\end{tabular} & $\begin{array}{llll}\mathrm{R} & \mathrm{R} & \mathrm{A} & + \\
\mathrm{C} & & + \\
\mathrm{R} & & & \\
\mathrm{R} & & \\
\end{array}$ & $\mathrm{R}+$ & \multirow{2}{*}{ Unzoned } \\
\hline $\begin{array}{l}7-2,10-12 \\
7-3,10-12 \\
7-5,10-12 \\
7-6,10-12 \\
7, \mathrm{CC}\end{array}$ & $\begin{array}{l}\mathrm{R} \\
\mathrm{F} \\
\mathrm{C}\end{array}$ & & $\begin{array}{llll}\mathrm{R} & + & & \\
\mathrm{F} & + & & \mathrm{R} \\
\mathrm{R} & \mathrm{R} & + & \mathrm{R} \\
\mathrm{F} & \mathrm{R} & & \\
\mathrm{F} & \mathrm{F} & \mathrm{R} & \mathrm{R} \\
\mathrm{F}\end{array}$ & $\begin{array}{l}+ \\
\mathrm{F} \\
\mathrm{R}\end{array}+$ & $1^{+}$ & + & $\begin{array}{l}++ \\
R\end{array}$ & $\begin{array}{l}++ \\
+ \\
+\end{array}$ & $\begin{array}{l}\mathrm{F}+ \\
\mathrm{A}+ \\
\mathrm{A} \\
\mathrm{A} \\
\mathrm{A}\end{array}$ & $\begin{array}{ll}\mathrm{R} & \\
\mathrm{C} & \mathrm{F} \\
\mathrm{C} & \mathrm{F} \\
\end{array}$ & 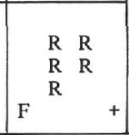 & \\
\hline
\end{tabular}

Note: $\mathrm{A}=$ abundant $; \mathrm{C}=\operatorname{common} ; \mathrm{F}=$ frequent $\mathrm{R}=\operatorname{rare} ; \mathrm{G}=\operatorname{good} ; \mathrm{M}=$ moderate.

Larnacantha polyacantha Campbell and Clark, 1944, pp. 30, 31, pl. 5, figures 4-7. (Plate 1, figure 10).

Lychnocanomma elongata (Vinassa), Sanfilippo and Riedel, in Sanfilippo et al., 1973, p. 221, pl. 5, figures 19, 20. = Tetrahedrina elongata Vinassa, 1900, p. 243, pl. 2, figure 31. (Plate 2, figure 12).

Phormostichoartus corbula (Harting), Nigrini, 1977, p. 252, pl. 1, figure $10=$ Lithocampe corbula Harting, 1863, p. 12, pl. 1, figure 21 .

Rhodospyris sp. cf. De-1 group, Petrushevskaya and Kozlova, 1972, p. 531, pl. 38, figures 15,16 (Plate 1, figure 12).

Siphocampe arachnea (Ehrenberg) group, Nigrini, 1977, p. 255, pl. 3, figure $7,8=$ Lithocampe lineata Ehrenberg, 1838, p. 130 (partim).

Sphaeropyle langii Dreyer, 1889, p. 13, pl. 4, figure 54.
Spirema sp., Kling, 1973, p. 635, pl. 7, figures 23-25. Remarks: Although not abundant, this taxon is continuously observed in the Miocene section of Hole 433A. (Plate 1, figure 9).

Sprirocyrtis subscalaris Nigrini, 1977, pp. 259, 260, pl. 3, figures 1,2 $=$ Lithamphora furcaspiculata Popofsky, 1913, pl. 408, textfigure 132 (partim). (Plate 2, figure 21).

Spongodiscus sp., Ling, 1973, p. 778, pl. 1, figures 9, 10. Remarks: Apparently this is the same taxon recognized from the Leg 19 materials. Occurrence in Hole 433A sediments extends their geological range downward to lower Pliocene. (Plate 1, figure 7).

Spongopyle osculosa Dryer, 1889, pp. 42, 43, pl. 11, figures 99, 100. Stichocorys delmontensis (Campbell and Clark), Sanfilippo and Riedel, 1970, p. 451, pl. 1, figure $9=$ Eucyrtidium delmontensis Campbell and Clark, 1944, p. 56, pl. 7, figures 19, 20. (Plate 2, figure 10). 
Stichocorys peregrina (Riedel), Sanfilippo and Riedel, 1970, pp. 451, 452 , pl. 1, figure 10 = Eucyrtidium elongatum peregrinum Riedel, 1953 , p. 812 , pl. 85 , figure 2 . (Plate 2, figure 11).

Stylatractus universus Hays, 1970, p. 215, pl. 1, figures 1, 2. (Plate 1, figure 2).

Stylochlamydium venustum (Bailey), Haeckel, 1887, p. $515=$ Perichlamidium venustum Vailey, 1856, p. 6, figures 16, 17. (Plate 1, figure 8).

Theocorys redondoensis (Campbell and Clark), Kling, 1973, p. 638, pl. 11, figures $26-28=$ Theocyrtis redondoensis Campbell and Clark, 1944, p. 49, pl. 7, figure 4. (Plate 2, figure 13).

Theocalyptra bicornis (Popofsky), Riedel, 1958, p. 240, pl. 4, figure 4 $=$ Theocorys bicornis Popofsky, 1908, pp. 288, 289, pl. 34, figures 7, 8. (Plate 2, figure 3 ).

Tholospyris cortinisca (Haeckel), Goll, 1969, pp. 325, 326, pl. 56, figures $3,5,6,8=$ Tripospyris (Tripospyrantha) cortiniscus Haeckel, 1887, p. 1026, pl. 84, figure 6. (Plate 1, figure 13).

Triceraspyris? sp., Ling et al., 1971, pp. 713, 714, pl. 2, figures 1-3; figure 7. Remarks: Like previous observations from the Leg 19 and the Bering Sea surface sediments, two different forms are grouped here provisionally. Their occurrence from Hole 433A extends the range to early Pliocene. Furthermore, the present observation confirms that a form without basal spines has slightly longer geological range than that with spine form. (Plate 1, figures $14,15)$.

\section{REFERENCES}

Bandy, O. L., Casey, R. E., and Wright, R. C., 1971, Late Neogene planktonic zonation, magnetic reversals, and ra- diometric dates, Antarctic to the Tropics. In Reid, J. L. (Ed.), Antarctic Oceanology I: Antarctic Research Series, v. 15, pp. 1-26.

Foreman, Helen P., 1975. Radiolaria from the North Pacific, Deep Sea Drilling Project, Leg 32. In Larson, R. L., Moberly, R., et al., Initial Reports of the Deep Sea Drilling Project, v. 32: Washington (U. S. Government Printing Office), pp. 579-676.

Hays, J. D., 1970. Stratigraphy and evolutionary trends of Radiolaria in North Pacific deep-sea sediments. In Hays, J. D. (Ed.), Geological Investigations of the North Pacific: Geol. Soc. Amer., Mem. 126, pp. 185-218.

Jackson, E. D., Koizumi, I., et al., 1978. Drilling confirms hot-spot origins, Geotimes, v. 23, no. 4, pp. 23-26.

Ling, H. Y., 1973. Radiolaria: Leg 19 of the Deep Sea Drilling Project. In Creager, J. S., Scholl, D. W., et al., Initial Reports of the Deep Sea Drilling Project, v. 19: Washington (U. S. Government Printing Office), pp. 777-797.

1975. Radiolaria: Leg 31 of the Deep Sea Drilling

Project. In Karig, E. D., Ingle, J. C., Jr., et al., Initial Reports of the Deep Sea Drilling Project, v. 31: Washington (U. S. Government Printing Office), pp. 703-761.

, 1979. Radiolarians from the west flank of Reykjanes Ridge, Leg 49 of the Deep Sea Drilling Project. In Luyendyk, B. P., Cann, J. R., et al., Initial Reports of the Deep Sea Drilling Project, v. 49: Washington (U. S. Government Printing Office), pp. 703-761. 
PLATE 1

(Magnification $\times 200$ unless otherwise indicated.)

Figure $1 \quad$ Druppatractus acquilonius 433A-1-1, 23-25 cm, R-2 (N25/1)

Figure 2 Stylatractus universus 433A-4, CC, R-2 (L31/0)

Figure 3 Cannartus prismaticus 433A-6, CC, R-1 (W10/4)

Figure 4 Cannartus violina 433A-6-7, 47-49 cm, R-1 (F24/4)

Figure $5 \quad$ Heliodiscus asteriscus 433A-6-4, 80-82 cm, R-1 (X13/4)

Figure 6 Euchitonia furcata 433A-6-4, 80-82 cm, R-1 (G17/4)

Figure $7 \quad$ Spongodiscus sp. 433A-2-1, 125-127 cm, R-1 (E9/4)

Figure 8 Stylochlamydium venustum 433-1-1, 75-77 cm, R-1 (E34/3)

Figure 9 Spirema sp. 433A-7-1, 80-82 cm, R-2 (G5/4)

Figure 10 Larnacantha polyacantha 433A-4-5, 10-12 cm, R-1 (K33/1)

Figure 11 Helotholus vema 433A-1-1, 70-73 cm, R-2 (Q14/4), ×250

Figure $12 \quad$ Rhodospyris sp. cf. De-1 group 433A-6-6, 140-142 cm, R-1 (W11/0), × 250

Figure 13 Tholospyris cortinisca 433A-7, CC, R-2 (J40/0), × 250

Figure 14 Triceraspyris ? sp. A. 433A-2, CC, R-2 (C32/4), × 250

Figure 15 Triceraspyris ? sp. B. 433A-2-2, 32-24 cm, R-1 (E25/2), ×250 
PLATE 1

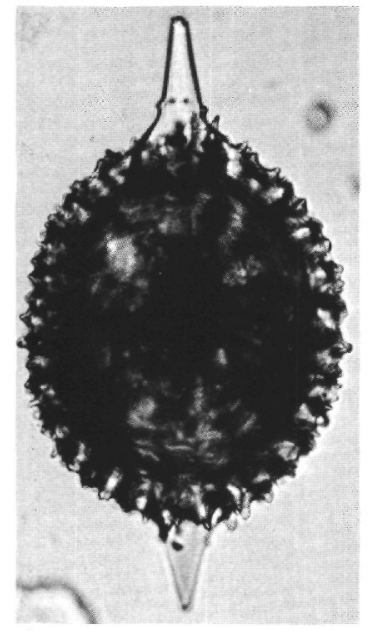

1
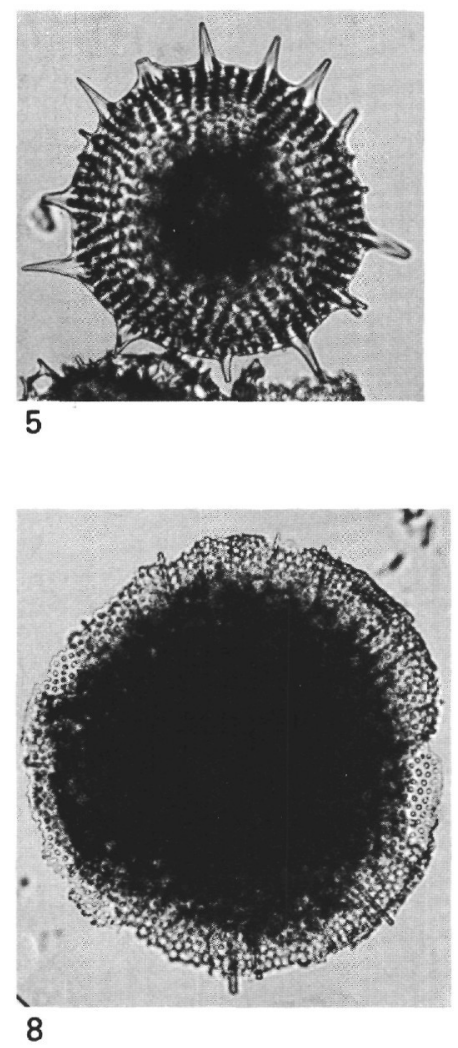
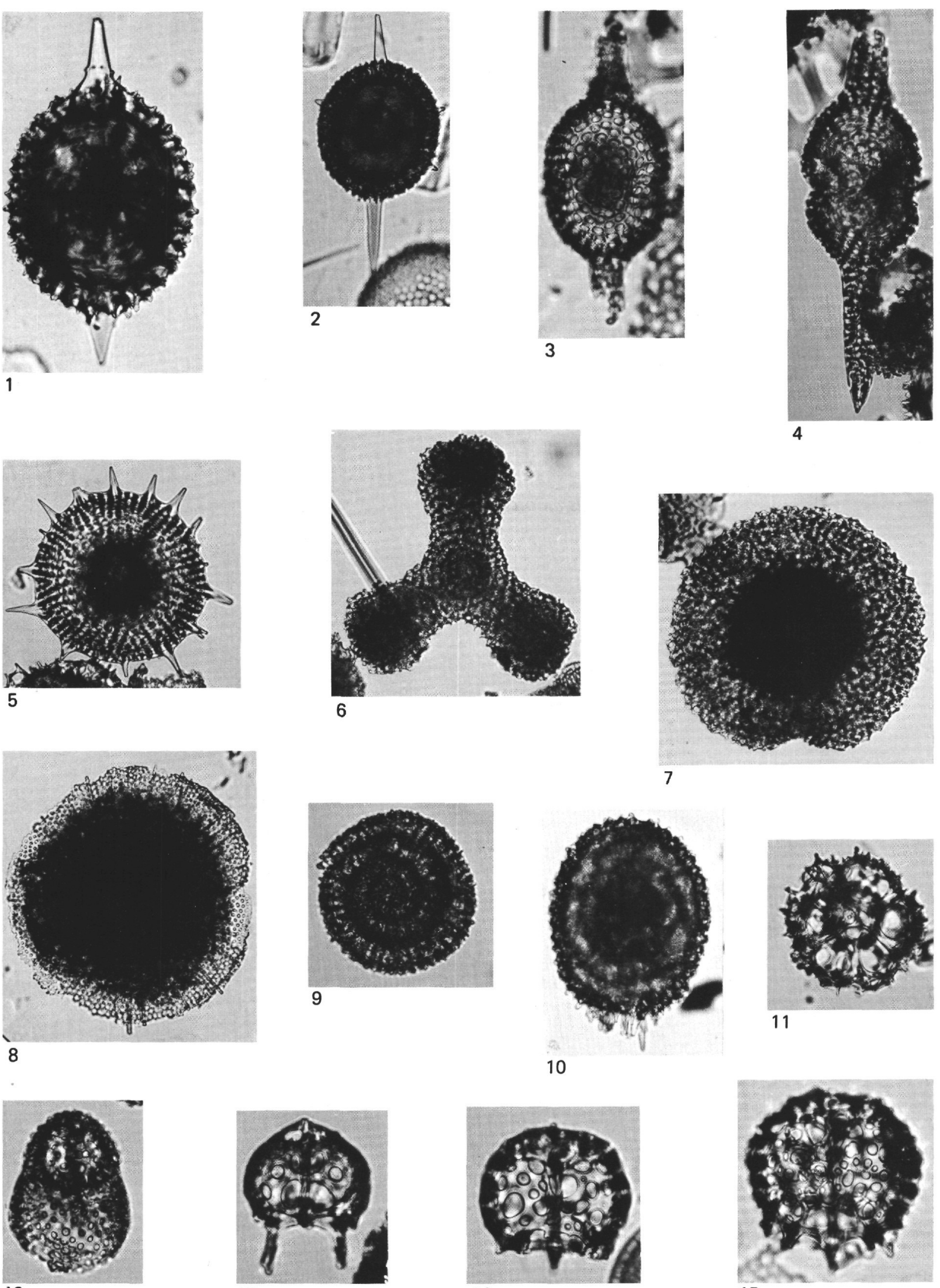

13
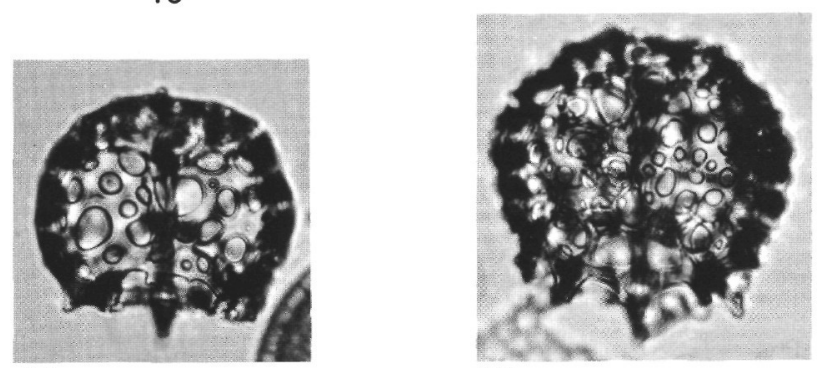

15 
PLATE 2

(Magnification $\times 200$ unless otherwise indicated.)

Figure 1 Cycladophora davisiana cornutoides 433A-6-6, 10-12 cm, R-1 (J33/3), $\times 250$

Figure 2 Clathrocyclas craspadota 433A-4-6, 10-12 cm, R-1 (L32/2)

Figure 3 Theocalyptra bicornis 433A-3-2, 13-15 cm, R-1 (R28/1)

Figure $4 \quad$ Cyrtocapsella japonica 433A-6-7, $47-49 \mathrm{~cm}, \mathrm{R}-1(\mathrm{~L} 27 / 3), \times 250$

Figure $5 \quad$ Cyrtocapsella tetrapera 433A-7-1, 80-82 cm, R-1 (E27/0)

Figure $6 \quad$ Cyrtocapsella sp. 433A-6-7, 47-49 cm, R-1 (V32/1)

Figure 7 Eucyrtidium calvertense 433A-4-1, 13-15 cm, R-2 (R5/2)

Figure 8 Eucyrtidium matuyamai $433-1-2,10-13 \mathrm{~cm}, \mathrm{R}-1$ (H10/1)

Figure 9 Eucyrtidium sp. 433A-7-3, 10-12 cm, R-1 (D27/0)

Figure $10 \quad$ Stichocorys delmontensis 433A-4-6, 10-12 cm, R-1 (K22/3)

Figure 11 Stichocorys peregrina 433A-6-6, 10-12 cm, R-1 (D31/0)

Figure 12 Lychnocanomma elongata 433A-7,CC, R-1 (D7/2)

Figure 13 Theocorys redondoensis 433A-4,CC, R-1 (D12/3)

Figure 14 Anthocyrtidium ophirense 433A-4,CC, R-1 (W2/1)

Figure 15 Lamprocyclas maritalis maritalis 433A-2,CC, R-2 (Y9/4)

Figure 16 Lamprocyclas sp. 433A-4-1, 13-15 cm, R-2 (S4/2)

Figure 17 Lamprocyrtis heteroporos 433A-1-1, 23-25 cm, R-2 (Q12/0)

Figure 18 Carpocanistrum sp. A 433A-7-5, 10-12 cm, R-2 (E21/2)

Figure 19 Carpocanistrum sp. C 433A-6,CC, R-1 (J17/1)

Figure 20 Botryostrobus aquilonaris 433-1-2, 10-13 cm, R-1 (Q8/0)

Figure $21 \quad$ Spirocyrtis subscalaris 433A-2,CC, R-2 (C25/1) 

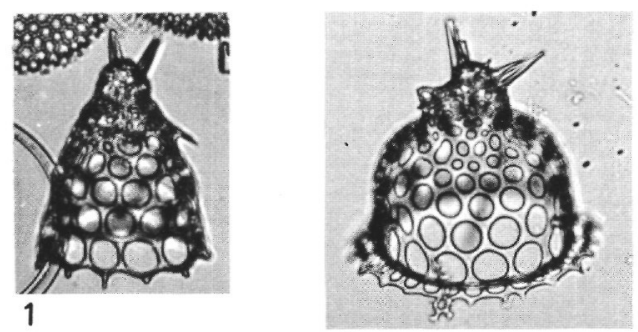

2
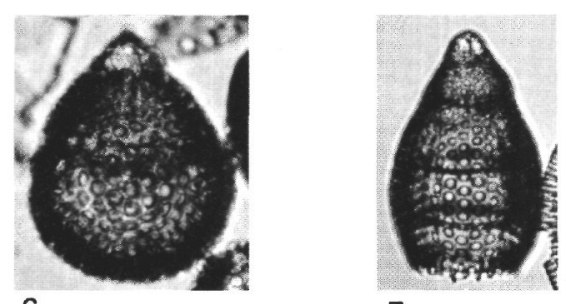

6
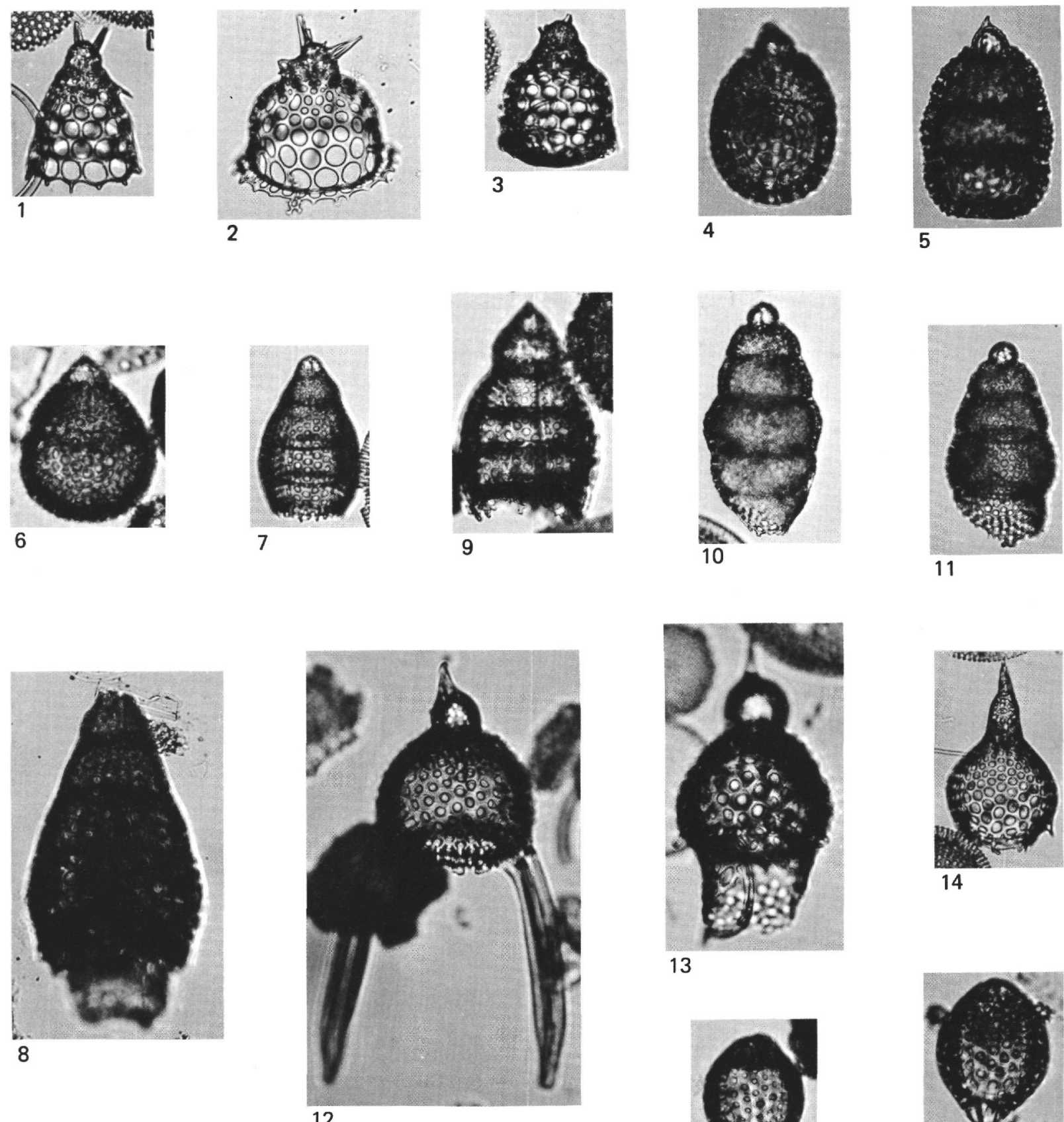

11
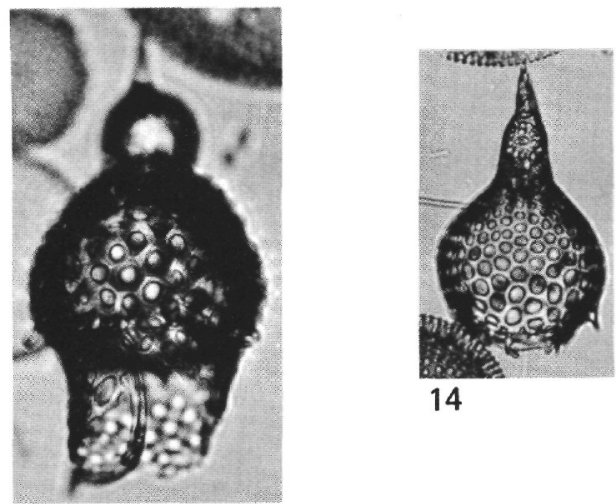

13

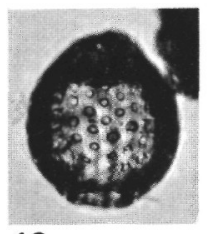

xख98\% 18

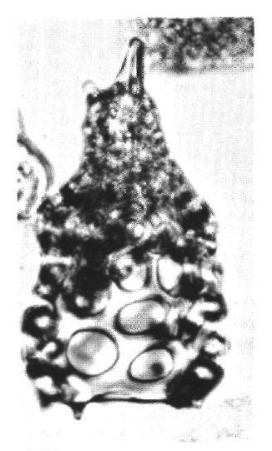

17
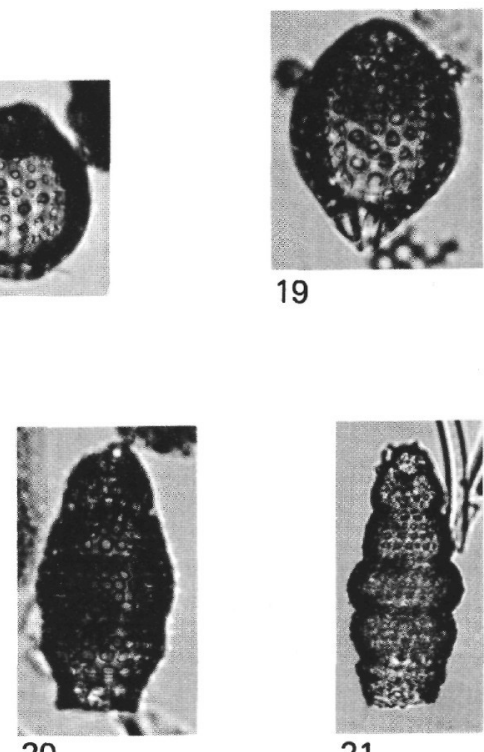

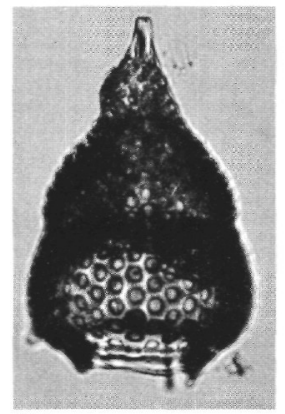

15

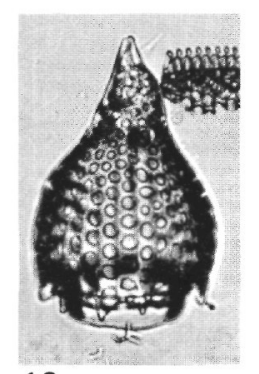

16
20

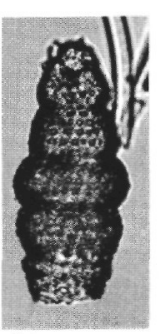

21 